\title{
A New Security Warning Model about Power Grid
}

\author{
HuiJing $\mathrm{Bi}^{1}$, HongYan Zhang ${ }^{1}$,YueMei Jiang ${ }^{2}$ and XiLan Zhao ${ }^{1}$ \\ ${ }^{1}$ State Grid Hebei Power Company, Training Centre, Shijiazhuang, China \\ ${ }^{2}$ Beijing Kedong Electric Control System Co., Ltd, Beijing, China \\ ${ }^{3}$ State Grid Gansu Electric Power Company, Dispatch department, Tianshui, China
}

\begin{abstract}
In recent years, lots of power grid blackouts at home and abroad indicates that the meteorological disasters caused by external meteorological conditions has gradually rose to major contradiction of power grid security. Basing on release information of meteorology, lightning monitoring information and power transmission equipment monitoring information, it established a weather warning model about power grid security, combined with real-time security analysis. Firstly, mathematical models of various types of weather conditions and weather risk assessment model grid were built, then actual operating conditions of a certain regional power grid and model results were compared, the comparison result prove the accuracy of the warning model, and provides a strong recommendation for decision-making.
\end{abstract}

\section{Introduction}

Meteorological natural disasters are the main factors threatening the safety of modern power grids. According to the statistics, meteorological disasters caused by the grid accident accounted for about $70 \%$ of the total number of accidents. In 2002, the United States, Missouri suffered raging snow disaster, resulting in nearly $40 \%$ of the $500 \mathrm{kV}$ transmission line outage. In 2008, China's southern rain and snow continued extreme weather, resulting in more than $10 \mathrm{kV}$ line parking up to 7500 , $35 \mathrm{kV}$ substation outage load of 40 million $\mathrm{kW}$, resulting in more than 33 million users out of power, 110 million people affected by electricity, direct economy Loss of more than 1500 million yuan. Therefore, extreme weather disasters on China's current and future safe operation of the power grid constitutes a serious threat[1-2].

In recent years, all levels of power companies to increase investment, efforts to enhance the weather forecast real-time, professional and fine degree[3-6]. At present, although some of our power dispatching center or power grid emergency command center to build the grid weather warning system[7-8], but these systems and technical solutions focus on meteorological information collection, monitoring, forecasting and early warning, in the security warning did not power grid real-time Safety analysis and the probability of meteorological disasters combined with the grid, did not achieve quantitative risk assessment. At the same time, these systems are not deep enough to study the outage model of power grid equipment for different meteorological disasters, and do not realize the accurate quantitative analysis, and the grid dispatching center still lacks effective early warning means for grid weather hazard monitoring. Therefore, this paper studies and builds the mathematical model of meteorological conditions and the meteorological risk assessment model of the grid, and uses the meteorological information of the meteorological department, the lightning monitoring information and the meteorological information of the power transmission equipment to establish the relationship between the meteorological model and the risk assessment model, For all types of meteorological conditions under the grid dispatch department defense and decision-making to find new ways.

\section{System structure and working principle}

The early warning system model consists of several modules: weather forecast sub-module, lightning detection information sub-module, transmission equipment detection system sub-module, grid state estimation sub-module, meteorological risk on-line analysis and early warning sub-module and meteorological risk result and alarm sub-module The As shown in Figure 1.

The early warning system first collects, integrates and processes various meteorological information, geographic information, power transmission and transformation equipment model, and realizes the above information and grid model, as well as the integration with the online running state, based on real-time steady-state power system data, Probability Analysis of Equipment Discontinuance for Weather Safety. Then, static safety 
risk analysis is carried out based on the static safety assessment index system of grid meteorological safety. Finally, the static safety assessment and early warning of power grid are carried out according to the grid risk assessment index.

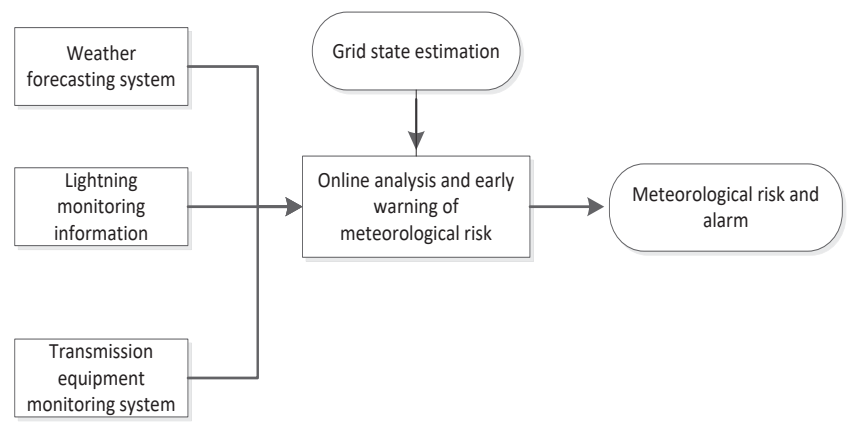

Figure 1. New grid weather weather warning model structure diagram

\section{Probabilistic model of power plant equipment outage}

\subsection{Probabilistic model of power plant equipment outage under lightning conditions}

In order to evaluate the accuracy of the thunder zone forecast, use $R_{\mathrm{POD}}$ (probability of detection, POD), $R_{\mathrm{FAR}}$ (false alarm ratio, FAR)and $R_{L D R}$ (lighting detection probability, LDP), As shown in (1) - (3):

$$
\begin{gathered}
R_{\mathrm{POD}}=\frac{E \cap A}{A} \times 100 \% \\
R_{\mathrm{FAR}}=\frac{E \cap \bar{A}}{E} \times 100 \% \\
R_{\mathrm{LDP}}=\frac{(E \cap A) \times \operatorname{Max}\left\{p^{\prime}, p\right\}}{A \times p} \times 100 \%
\end{gathered}
$$

In the formula, $E$ is the area of the predicted lightning area; $A$ is the actual lightning area; $\bar{A}$ is the actual area of non-lightning area; $E \cap A$ is the area of lightning area that is accurate; $E \cap \bar{A}$ is the area of lightning area predicted by mistake; $p^{\prime}$ is the predicted lightning density; $p$ is the actual lightning density; $E \cap A \times \max \left\{p^{\prime}, p\right\}$ is the number of successful mines; $A \times p$ is the actual lightning strikes.

\subsection{Probability model of power equipment outage under wind condition}

In the air pressure, rainfall, temperature, relative humidity and many other micro-meteorological factors, the greatest threat to the power grid is caused by the wind caused by the wind flashover. The wind speed at the time of wind flashover is random and can be used to model the probability of airborne outage.

The transmission line design specification specifies the minimum gap $d_{c}$ between the live part and the tower component under the corresponding wind conditions. When the wind speed $v$ is much smaller than the critical wind speed $v_{c}$, the wind angle is smaller and the minimum air gap will be much larger than the minimum gap $d_{c}$, so the line will not occur wind partial flashover, that is, the probability of wind bias trip $P_{M}$ is 0 ; when the wind speed $v$ is much larger than the critical wind speed $v_{c}$, the wind angle is large, the minimum air gap will be far less than the minimum gap $d_{c}$, so the line will occur wind flash, that is, the probability of wind bias trip $P_{M}$ is 1 ; when the wind speed $v$ is near the critical wind speed $v_{c}$, the probability of wind deviation $P_{M}$ is between $(0,1)$, and the same trend as wind speed. In order to make the outage probability model more simple and practical, we use the piecewise linear model approximation as shown in Figure 2.

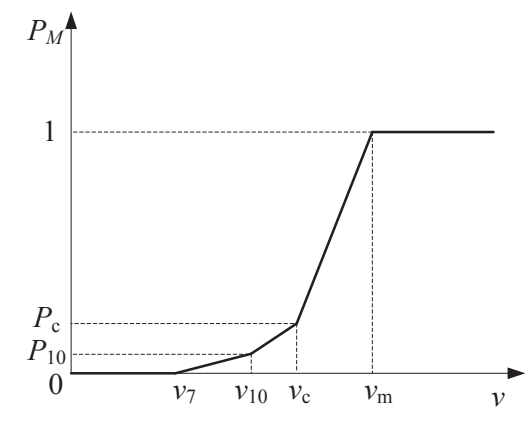

Figure 2. transmission line wind offset device model

Figure 2 in the wind speed turning points $v_{7} 、 v_{10}$ were 7 and 10 wind speed, $v_{c}$ is critical wind speed of the line, $P_{7}, P_{10}$ and $P_{c}$ are the probability of outage at the corresponding wind speed, $v_{m}$ is the minimum wind speed that must occur for tripping, the probability of outage is 1 . $P_{10}, P_{c}, v_{c}$ and $v_{m}$ can be obtained by linear fitting of the wind speed data of the grid micro-meteorological monitoring equipment and the wind-skew trip accident record.

\subsection{Probability model of power plant equipment outage under typhoon condition}

Typhoon is one of the major catastrophic weather that affects our coastal areas and some inland areas. The probability model of typhoon and wind deviation is based on wind speed as the main influencing factor, and the same model can be used.

According to the latitude and longitude coordinates of the tower, the tower towers within the influence range of the typhoon are selected, and the typhoon forecast wind speed is the main influencing factors. According to the probability model of the wind line deviation of the transmission line, the typhoon outage probability of the tower is calculated. Finally, the typhoon outage probability of each tower is calculated and the typhoon outage probability of the transmission line is calculated.

$$
P_{Y}=1-\prod_{i=1}^{n}\left(1-P_{Y i}\right)
$$

Where $P_{Y}$ is the typhoon outage probability of the line; $n$ is the number of towers for the line; $P_{Y i}$ is the typhoon outage probability of the $i$-th pole tower. 


\subsection{The Probability Model of Equipment Outage in Integrated Meteorological Condition}

Let the transmission line $i$ lightning outage probability $P_{L i}$, micro-meteorological outage probability $P_{M i}$, typhoon outage probability $P_{Y i}$. The micro-meteorological and typhoon outage probabilistic models take the wind speed as the main influencing factor, so the two take the maximum value as the wind outage probability $P_{W i}$, ie

$$
P_{W i}=\max \left(P_{M i}, P_{Y i}\right)
$$

The probability of lightning outage and the probability of wind outage are the logical series relationship, the probability of the total meteorological outage of the transmission line $i$ is

$$
P_{\mathrm{T} i}=1-\left(1-P_{L i}\right)\left(1-P_{W i}\right)
$$

\section{Meteorological risk assessment model for power grid}

The meteorological conditions of the components (transmission lines, transformers) of the grid are usually not the same during meteorological disasters (such as lightning, wind), so the probability of failure (outage) is different and the probability of the state of the grid is different, The corresponding operational risk is not the same. The purpose of the operational risk assessment is to find out the risk of the system operating state, that is, its probability and consequences of a larger system state, and the system state according to the size of the risk indicators in descending order for the dispatcher to provide the current risk of large systems status information.

Assuming that $P_{0 j}$ and $P_{1 j}(t)$ are the probability that the component $j$ is in the run and outage state at time $t$, ie, $P_{0 j}(t)+P_{1 j}(t)=1$, the outage probability $P_{1 j}(t)$ can be calculated from the meteorological outage probability.

Let the state of the system state $S$, in the running state of the composition of the composition $C_{0}$, in the outage state of the components constitute the set $C_{1}$, then $t$ time system state $S$ probability can be calculated using the following formula

$$
P_{S}=\prod_{j \in C_{0}} P_{0 j}(t) \cdot \prod_{j \in C_{1}} P_{1 j}(t)
$$

The probability of all system states can be calculated by equation (5). If the number of elements in the grid is $N$, the total number of system states $N_{T}=2^{N}$, if $N$ is large, then $N_{T}$ is very large and the risk assessment of all system states is In this paper, we consider only the system state of the number of outage components $N_{f} \leqslant 2$, and select the top probability of the top $N_{S}$ system states from the $N_{T}$ system states for risk assessment.

The operating risk index of any system state $S$ in the top probability of the top $N_{S}$ system states from the maximum system state is:

$$
\operatorname{Risk}(S)=P_{S} \cdot \operatorname{Sev}(S)
$$

Where $\operatorname{Risk}(S)$ is the operational risk index of the system state $S, P_{S}$ is the probability of the system state $S$, obtained from the meteorological outage probability calculation model, $\operatorname{Sev}(S)$ is the consequence of the system state $S$, and the consequences reflect the severity of the outage failure in the system state, "Consequences" is very broad, can be the degree of line overload, the degree of bus voltage limit, the degree of cross-section of the trend and so on. Define the consequences according to the scope of the assessment. According to the definition of the scope of the assessment of the consequences of the risk assessment indicators can be divided into two categories, one is the system risk assessment indicators, and the other is the cross-sectional risk assessment indicators.

\subsection{System risk assessment index and system risk alarm}

The early warning model uses the MSFS (margin of system flow security) as the evaluation index, the specific expression is as follows:

$$
\operatorname{MSFS}_{S}=P_{S} \cdot \min _{a \in A}\left(1-\frac{L_{a}}{L_{a, \text { max }}}\right)
$$

Where: $\mathrm{MSFS}_{S}$ is the total safety margin of all line currents in the system if there is no line overload in the system state $S ; P_{S}$ is the probability of the system state $S$; $L_{a}$ is the trend of line $a, L_{a, \max }$ is the maximum allowed for line $a$ transmit active power; $A$ is the line set in the system.

Because all the selected system state must be a power flow calculation, if you need to assess the system state is very large, the calculation is quite large. Because these system states are based on the ground state on the basis of one or more line outage (breaking fault), in order to improve the calculation speed, it can be broken through the DC method to calculate the power flow $L_{a}$. DC method to break the flow of the calculation of the use of ground state power flow of the DC node charge matrix $B_{0}$ factor table, do not re-form the factor table, can greatly speed up the breaking calculation.

$$
\operatorname{MSVS}_{S}=\min \left\{\mathrm{MSHVS}_{S}, \mathrm{MSLVS}_{S}\right\}
$$

Where $\mathrm{MSVS}_{S}$ is the total safety margin of all bus voltages in the system if there is no bus voltage in the system state $S$; $\mathrm{MSHVS}_{S}$ is the upper limit safety margin of the system voltage, $\mathrm{MSLVS}_{S}$ is the lower limit of the system voltage margin, and its definition (9), (10), so $M_{S V S}$ should take the minimum value of $M_{S H V S}$ and MSLVS $_{S}$.

$$
\begin{aligned}
& \operatorname{MSHVS}_{S}=P_{S} \cdot \min _{b \in B}\left(1-\frac{V_{b}}{V_{b, \text { max }}}\right) \\
& \operatorname{MSLVS}_{S}=P_{S} \cdot \min _{b \in B}\left(\frac{V_{b}}{V_{b, \min }}-1\right)
\end{aligned}
$$

In the above formula: $V_{b}$ for the bus $b$ voltage; $V_{b, \max }$ for the bus $b$ voltage upper limit; $V_{b, \min }$ for the bus $b$ voltage lower limit; $B$ for the system bus set.

It is not possible to give the information of the amplitude of the bus voltage, but the fast decomposition method based on the compensation principle is used to calculate the current flow. Matrix B' and $\mathbf{B}^{\prime \prime}$ factor table, do not re-form the factor table, you can quickly calculate the voltage $V_{b}$. 


$$
\mathrm{DSO}_{S}=P_{S} \cdot \max _{a \in A}\left(\frac{L_{a}}{L_{a, \max }}-1\right)
$$

Where $\mathrm{DSO}_{S}$ is the total overload risk of all line currents in the system if there is a line overload in the system state $S$, and the consequence is the overload level of the most overloaded lines in all overload lines; $A$ is the set of overload lines in the system The If there is no line overload, then $\mathrm{DSO}_{S}$ is 0 .

\subsection{Section risk assessment index and section risk warning}

Margin of flow-gate flow security (MFFS) calculation formula is as follows:

$$
\operatorname{MFFS}_{S}=P_{S} \cdot\left(1-\frac{\sum_{a \in F A} L_{a}}{L_{F, \text { max }}}\right)
$$

Where $\mathrm{MFFS}_{S}$ is the total safety margin for all line currents in section $F$ in the cross section of system state $S ; L_{F, \text { max }}$ is the maximum transmission power limit for section $F ; F A$ is the line set in section $F$.

Degree of flow-gate overload (DFO) calculation formula is as follows:

$$
\mathrm{DFO}_{S}=P_{S} \cdot\left(\frac{\sum_{a \in F A^{\prime}} L_{a}}{L_{F, \text { max }}}-1\right)
$$

Where $\mathrm{DFO}_{S}$ is the total overloaded power of the active power transmitted in section $F$, i.e., the overload of the transmission power of the section $F$, if the active power transmitted by section $F$ exceeds the transmission power limit in system state $S ; F A^{\prime}$ is the set of lines that are overloaded in the section $F$.

\subsection{Operational risk assessment index calculation}

Based on the above operational risk assessment indicators, the calculation of the operational risk assessment indicators, calculation and calculation of the system alarm can be shown in Figure 3 .

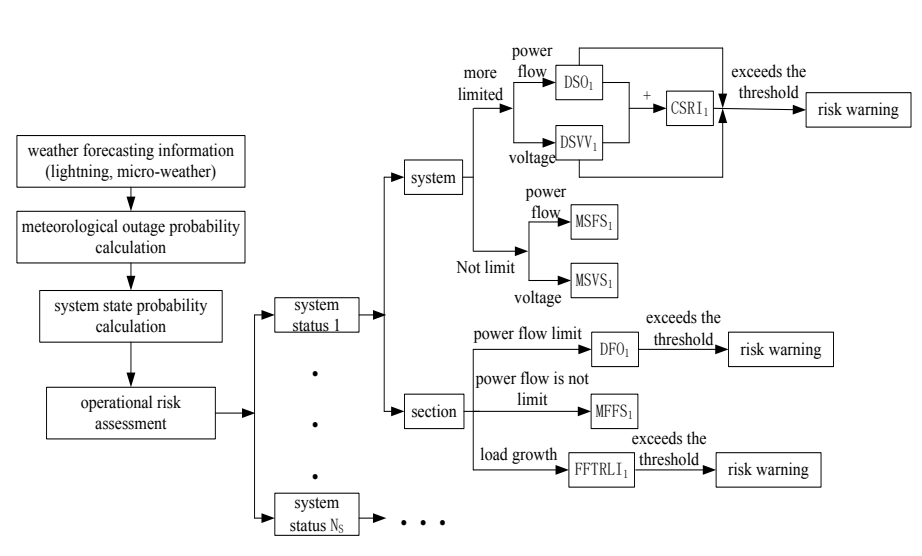

Figure 3. Operational risk assessment index calculation

\section{System operation example}

In order to verify the accuracy and practicability of the model, this paper takes the relevant data of a certain power grid as an example to detect. The weather data is provided by the grid weather station and transmitted through the network to the meteorological information server database of the dispatching automation system area III. Through the development of the interface program, you can directly access the meteorological information database, and regularly extract the relevant weather information.

Part of the data for the December 2013 period of the grid was predicted using the method of 1 minute ahead of the line lightning strikeout (the results are shown in Table 1.). The actual occurrence of 12 lightning accident occurred in the grid, of which three times due to a large number of data missing, cannot be calculated for the probability of outage can be calculated 9 outage accident occurred in the $220 \mathrm{kV}$ or $500 \mathrm{kV}$ line, based on this method can In this paper, the probability of stopping the outage line relative to the other lines in the minefield is indeed high (basically all in the forefront), and the validity and correctness of the method are proved again.

Table 1. Probability prediction of power outage

\begin{tabular}{|c|c|l|c|c|c|}
\hline $\begin{array}{c}\text { Fault } \\
\text { time }\end{array}$ & $\begin{array}{c}\text { Fault line } \\
\text { voltage } \\
\text { level(kV) }\end{array}$ & \multicolumn{1}{|c|}{ Fault line name } & Forecast results & $\begin{array}{c}\text { Predict the probability } \\
\text { of lightning strikeout }\end{array}$ & $\begin{array}{c}\text { Line outage probability } \\
\text { sort / mine area line } \\
\text { number }\end{array}$ \\
\hline $14: 43: 00$ & 220 & Line YiZao 4884 & success & 0.567176 & $1 / 4$ \\
\hline $17: 52: 00$ & 220 & Line NingQian 2D91 & success & 0.452720 & $3 / 5$ \\
\hline $17: 29: 00$ & 110 & Line LiFu 5941 & unpredictable & - & - \\
\hline $15: 15: 00$ & 500 & Line ShanLi 5366 & success & 0.2202 & $5 / 6$ \\
\hline $16.18: 00$ & 110 & $\begin{array}{l}\text { Line QingNing 481 /line } \\
\text { QingNing 482 }\end{array}$ & unpredictable & - & - \\
\hline $18: 15: 00$ & 500 & Line FangZhu 2873 & success & 0.913716 & $1 / 5$ \\
\hline $14: 48: 00$ & 500 & Line DuShan 5392 & success & 0.769957 & $1 / 7$ \\
\hline $16: 21: 00$ & 220 & Line DuTian 4868 & success & 0.376876 & $2 / 2$ \\
\hline $19: 52: 00$ & \pm 500 & Line NingQian 2D91 & unpredictable & - & - \\
\hline $00: 59: 00$ & 500 & Line FanHui 5904 & success & 0.988842 & $1 / 6$ \\
\hline $21: 09: 00$ & 220 & Line HanMiao 4846 & success & 0.698580 & $2 / 4$ \\
\hline $13: 26: 00$ & 220 & Line GeTian 2830 & success & 0.619922 & \\
\hline
\end{tabular}


The power grid $220 \mathrm{kV}$ transformer equivalent to the high side, and $500 \mathrm{kV}$ connection line and $1000 \mathrm{kV}$ UHV line outside the end as a balance node, a total of 389 nodes, 470 branches; power generation total output power of $21467 \mathrm{MW}$, The load of the province is $13635 \mathrm{MW}$, the provincial delivery of about $7800 \mathrm{MW}$.

The power flow in the ground state can be obtained by calculating the power flow in the state of the system running normally. The trend of all branches in the ground state is not limited by the power flow calculation. 47 dynamic sections were identified by the dynamic section identification program. It is shown that there are two sections of the cross-section of the trend, respectively, by the $220 \mathrm{kV}$ ZhenGang 2D55 line and the ZhenGang 2D56 line composed of two lines 1 ; by the $220 \mathrm{kV}$ ZhuZhen 4841 line and ZhuZhen 4842 line composed of two lines The limit of the cross section trend is shown in Table 2.

Table 2. Cross section trend limit

\begin{tabular}{|c|c|c|c|c|}
\hline No. & $\begin{array}{c}\text { Section } \\
\text { actual } \\
\text { power }\end{array}$ & $\begin{array}{c}\text { Transmission } \\
\text { power limit }\end{array}$ & $\begin{array}{c}\text { Cross } \\
\text { section } \\
\text { trend } \\
\text { limit }\end{array}$ & $\begin{array}{c}\text { Trend } \\
\text { limit } \\
\text { percentage }\end{array}$ \\
\hline 1 & $505 \mathrm{MW}$ & $318 \mathrm{MW}$ & $187 \mathrm{MW}$ & $58.9 \%$ \\
\hline 2 & $376 \mathrm{MW}$ & $341 \mathrm{MW}$ & $35 \mathrm{MW}$ & $10.3 \%$ \\
\hline
\end{tabular}

As can be seen from Table 2, the trend of the crosssection of the maximum percentage of the limit, the most serious limit.

The probability of occurrence of meteorological factors and their outages is shown in Table 3. The probability of outage in the table indicates the total probability of meteorological outage for each return line in the double-circuit line, the probability of meteorological outage for each return line in the return line is equal.

Table 3. The probability of outage of each branch of the system

\begin{tabular}{|c|c|c|}
\hline Branch & $\begin{array}{c}\text { Affect meteorological } \\
\text { factors }\end{array}$ & $\begin{array}{c}\text { Outage } \\
\text { probability }\end{array}$ \\
\hline $\begin{array}{c}\text { YuChen 4723/4727 } \\
\text { line }\end{array}$ & Lightning & 0.16 \\
\hline $\begin{array}{c}\text { ShuangDeng 4C85 } \\
\text { line }\end{array}$ & typhoon & 0.067 \\
\hline $\begin{array}{c}\text { ShuangAn } \\
\text { 4C81/4C82 line }\end{array}$ & typhoon & 0.11 \\
\hline LuoGu 2768 line & Micro meteorology & 0.4 \\
\hline
\end{tabular}

When calculating the risk value of the grid, the consequence $C$ uses the cumulative value of the limit of the branch flow and the limit of the cross-section trend to characterize the total trend of the system. For the sake of space, only the first five branches of the system with the largest risk of failure are listed, as shown in Table 4.

Table 4. Fault risk value

\begin{tabular}{|c|c|c|c|}
\hline $\begin{array}{c}\text { Fault } \\
\text { number }\end{array}$ & $\begin{array}{c}\text { Fault } \\
\text { probability }\end{array}$ & Stop the branch & $\begin{array}{c}\text { Risk } \\
\text { value }\end{array}$ \\
\hline 1 & $2.78 \times 10^{-1}$ & LuoGu 2768 line & $61.62 \mathrm{MW}$ \\
\hline 2 & $7.94 \times 10^{-2}$ & YuChen 4723 line & $17.57 \mathrm{MW}$ \\
\hline 3 & $5.29 \times 10^{-2}$ & $\begin{array}{c}\text { LuoGu } \\
2768 / \text { YuChen } 4723 \\
\text { line }\end{array}$ & $\begin{array}{c}11.67 \\
\text { MW }\end{array}$ \\
\hline 4 & $5.16 \times 10^{-2}$ & ShuangAn $4 \mathrm{C} 81$ line & $11.47 \mathrm{MW}$ \\
\hline
\end{tabular}

\begin{tabular}{|c|c|c|c|}
\hline 5 & $3.44 \times 10^{-2}$ & $\begin{array}{c}\text { LuoGu } \\
2768 / \text { ShuangAn } \\
\text { 4C81 line }\end{array}$ & $7.62 \mathrm{MW}$ \\
\hline
\end{tabular}

It can be seen from Table 4 . that the risk value of the "LuoGu 2768 line" outage failure is the largest and the probability of failure is the largest. The risk value exceeds the yellow warning threshold (30MW), and the yellow risk alarm should be given; the risk value of other faults Smaller than the blue warning threshold (0), should be given a blue risk alarm.

\section{Analysis conclusion}

Based on the meteorological information of the Bureau of Meteorology, the monitoring information of lightning and the monitoring information of transmission equipment, this paper establishes the model of grid safety weather warning based on real-time safety analysis of power grid. And the actual operation status of a provincial power grid is compared with the model calculation result. The comparison result shows the accuracy of the early warning model and provides a strong suggestion for the decision. In addition, the grid risk and safety evaluation index system is the technical problem of power supply control and operation decision support. The static risk safety evaluation index of grid in this project still needs to be tested, adjusted and perfected in practice, and based on long-term operation Experience to further develop other risk factors outside the meteorological factors, and ultimately establish a comprehensive grid safety and safety evaluation index system.

\section{References}

1. Yin Yonghua, Guo Jianbo, Zhao Jianjun, et a1. "Preliminary analysis of large scale blackout in interconnected North America power on August 14 and lessons to be drawn," Power System Technology, vol. 27, pp. 8-11 (2003).

2. Chen Yonjin, Ren Xia, Huang Wenying. "Model and analysis of power system reliability evaluation considering weather change," Automation of Electric Power Systems, vol. 28, pp. 17-21 (2004).

3. LIU Wengying, YANG Nan, ZHANG Jianli. "Complex Grid Failure Propagating Chain Model in Consideration of Adverse Weather," Proceedings of the CSEE, vol. 32, pp. 53-59(2012).

4. LuoYi, WangYingying, WanWei, et a1. "Fault chains model for cascading failure of gfid," Automation of Electric Power Systems, vol.33, pp. 15(2009).

5. Yu Qun, Guo Jianbo. "Statisics and self-organized criticality characters of blackouts in China electric power systems," Automation of Electric Power Systems, vol. 30, pp.16-21(2006).

6. Cao Yijia, Jiang Quanyuan, Ding Lijie. "Selforganized criticality phenomenon for power system blackouts," Power System Technology, vol. 29, pp.15(2005). 
7. Ding Ming, Han Pingping. "Research on power system cascading failure with complex system theory," Journal of Hefei University of Technology: Natural Science, vol. 28, pp.1047-1052(2005).

8. Wang Jiaming, Liu Wenying, Zhang Jianli. "Online Early Warning Model for Cascading Failure in
Complex Power Grid Under Severe Weather", Power System Technology, vol.36, pp.239-244(2012). 